\title{
Analytical Solution of Van Der Pol's Differential Equation Using Homotopy Perturbation Method
}

\author{
Md. Mamun-Ur-Rashid Khan \\ Department of Mathematics, University of Dhaka, Dhaka, Bangladesh \\ Email:mamun.math@du.ac.bd
}

How to cite this paper: Khan, Md.M.-U.-R. (2019) Analytical Solution of Van Der Pol's Differential Equation Using Homotopy Perturbation Method. Journal of Applied Mathematics and Physics, 7, 1-12. https://doi.org/10.4236/jamp.2019.71001

Received: November 20, 2018

Accepted: January 4, 2019

Published: January 7, 2019

Copyright (c) 2019 by author(s) and Scientific Research Publishing Inc. This work is licensed under the Creative Commons Attribution International License (CC BY 4.0).

http://creativecommons.org/licenses/by/4.0/

(c) (i) Open Access

\begin{abstract}
In this research work, Homotopy perturbation method (HPM) is applied to find the approximate solution of the Van der Pol Differential equation (VDPDE), which is a well-known nonlinear ODE. Firstly, the approximate solution of Van Der Pol equation is developed using Dirichlet boundary conditions. Then a comparison between the present results and previously published results is presented and a good agreement is observed. Finally, HPM method is applied to find the approximate solution of VDPDE with Robin and Neumann boundary conditions.
\end{abstract}

\section{Keywords}

Homotopy Perturbation Method (HPM), Van Der Pol Differential Equation (VDPDE), Nonlinear Differential Equations, Analytic Solution,

Boundary Conditions

\section{Introduction}

The Van Der Pol differential equation [1] can be written as

$$
y^{\prime \prime}+u\left(y^{2}-1\right) y^{\prime}+y=0
$$

where, $u$ is a scalar parameter. It is considered as an example of an oscillator with nonlinear damping, energy being dissipated at large amplitudes and generated as low amplitude. Such systems typically possess limit cycles: sustained oscillations around a state at which energy generation and dissipation balance [1] [2]. The main application by VDP models an electric circuit with a triode valve, the resistive properties of which change with current, the low current, negative resistance becoming positive as current increases. This model has been widely applied in science and engineering [3]. The parameter $u$ is indicating the nonlinearity and the strength of the damping. 
The concept of HPM was first established by He [4] [5], which has been used to solve a large number of non-linear problems. It is found that such approximations rapidly converges to the exact solution [4] [5]. Since it is not easy to find the solution of Equation (1.1) by usual methods such as the perturbation method, separation of variables etc., HPM can be used to find the analytical solution of nonlinear differential equations with different types of initial and boundary conditions [6] [7] [8] [9]. "In addition, the merging of perturbation method and the homotopy method is called homotopy perturbation method [4] [5], which has banished the deficiency of the traditional perturbation methods. On the other hand, this method can use the full benefit of perturbation techniques."

\section{Formulation of HPM}

The details of the following formulation is given in He which is presented below:

Consider the following non-linear differential equation [5]:

$$
M(y)-q(x)=0, x \in \phi
$$

with

$$
N\left(y, \frac{\partial y}{\partial n}\right)=0, x \in \Phi
$$

Here, $M$ has two parts (linear and nonlinear), $L n$ and NIn respectively. Then re-write Equation (2.1) as

$$
\operatorname{Ln}(y)+N \ln (y)-q(x)=0
$$

Then He [4] [5] introduced a homotopy $g(r, t): \phi \times[0,1] \rightarrow \mathbb{R}$ which satisfy

$$
H(g, t)=(1-t)\left[\operatorname{Ln}(g)-\operatorname{Ln}\left(v_{0}\right)\right]+t[M(g)-q(x)]=0, t \in[0,1], x \in \phi
$$

which is equivalent to

$$
H(g, t)=\operatorname{Ln}(g)-\operatorname{Ln}\left(v_{0}\right)+t \operatorname{Ln}\left(v_{0}\right)+t[\operatorname{Nln}(g)-s(x)]=0
$$

It follows from Equation (2.4a) and Equation (2.4b) that

$$
\begin{gathered}
H(g, 0)=\operatorname{Ln}(g)-\operatorname{Lln}\left(v_{0}\right)=0 \\
H(g, 1)=R(g)-q(x)=0
\end{gathered}
$$

"Thus, the changing process of $t$ from zero to unity is just that of $g(x, t)$ from $v_{0}(x)$ to $g(x)$. In topology, this is called deformation, and $\operatorname{Ln}(g)-\operatorname{Lln}\left(v_{0}\right), \quad M(g)-q(x)$ are called homotopic.” [4] [5]

Here, $t$ is very small and assume that

$$
g=g_{0}+\operatorname{tg}_{1}+t^{2} g_{2}+\cdots
$$

Setting $t=1$, approximate solution of Equation (2.1) can be obtained as,

$$
g=g_{0}+g_{1}+g_{2}+\cdots
$$

The convergence of series Equation (2.8) has been proved by He [4] [5] in his paper. 


\section{Numerical Examples}

In Example 1, the VDPDE with Dirichlet boundary conditions is solved by HPM and comparisons between the HPM and exact solutions are presented here after.

\section{Example 1:}

$$
y^{\prime \prime}+u\left(y^{2}-1\right) y^{\prime}+y=0, \quad y(0)=0, \quad y(20)=1
$$

Considering $y^{\prime \prime}+y$ as the linear part and $u\left(y^{2}-1\right) y^{\prime}$ as the nonlinear part we get the following Homotopy,

$$
Y^{\prime \prime}+Y-\left(y_{0}^{\prime \prime}+y_{0}\right)+t\left(y_{0}^{\prime \prime}-y_{0}\right)+t\left[u\left(Y^{2}-1\right) Y^{\prime}\right]=0
$$

Putting,

$$
Y=Y_{0}+t Y_{1}+t^{2} Y_{2}, y_{0}=\csc [20] \sin [x],
$$

in Equation (3.1) and equating the coefficients of $t$ from both sides, we get

$$
\begin{gathered}
y_{0}^{\prime \prime}+y_{0}=0, \quad y_{0}(0)=0, \quad y_{0}(20)=1 \\
y_{1}^{\prime \prime}+y_{1}+u y_{0}^{2} y_{0}^{\prime}-u y_{0}^{\prime}=0, \quad y_{1}(0)=0, \quad y_{1}(20)=0 \\
y_{2}^{\prime \prime}+y_{2}+2 u y_{0} y_{1} y_{0}^{\prime}-u y_{1}^{\prime}+u y_{0}^{2} y_{1}^{\prime}=0, \quad y_{2}(0)=0, \quad y_{2}(20)=0
\end{gathered}
$$

Solving we get,

$$
\begin{aligned}
y_{0}=\csc [20] \sin [x] \\
y_{1}=\frac{1}{16} u \csc [20]^{3} \sin [x](-40+x(2-4 \cos [40]) \\
+80 \cos [40]-\sin [40]+\sin [2 x]) \\
y_{2}=\left(\frac{1}{3072}\right) u^{2} \csc [20]^{3}(-18 \cos [5 x] \cot [20] \\
+144 \cos [20]^{2} \cos [40] \sin [x]-960 \cot [20] \sin [x] \\
+48 x \cot [20] \sin [x]-24 \cot [20]^{2} \sin [x] \\
-12 \cos [40] \cot [20]^{2} \sin [x]-12 \cos [80] \cot [20]^{2} \sin [x] \\
-9600 \csc [20]^{2} \sin [x]+960 x \csc [20]^{2} \sin [x] \\
-24 x^{2} \csc [20]^{2} \sin [x]-18 \cos [40] \csc [20]^{2} \sin [x] \\
+38400 \cos [40]^{2} \csc [20]^{2} \sin [x]-3840 x \cos [40]^{2} \csc [20]^{2} \sin [x] \\
+96 x^{2} \cos [40]^{2} \csc [20]^{2} \sin [x]+9 \cos [80] \csc [20]^{2} \sin [x] \\
+10 \cos [120] \csc [20]^{2} \sin [x]+12(7+4 \cos [40] \\
+4 \cos [80]) \cos [x]^{2} \csc [20]^{2} \sin [x] \\
+18 \cos [2 x] \csc [20]^{2} \sin [x]-24 \cos [80] \cos [2 x] \csc [20]^{2} \sin [x] \\
+ \\
+9 \cos [4 x] \csc [20]^{2} \sin [x]+24 \cos [40] \cos [4 x] \csc [20]^{2} \sin [x] \\
-10 \cos [6 x] \csc [20]^{2} \sin [x]+2160 \cot [20] \csc [20]^{2} \sin [x] \\
+1920 \cos [80] \cot [20] \csc [20]^{2} \sin [x]+48 \sin [40]^{2} \sin [x] \\
-480 \csc [20]^{2} \sin [80] \sin [x]+24 x \csc [20]^{2} \sin [80] \sin [x]
\end{aligned}
$$




$$
\begin{aligned}
& -240 \csc [20]^{4} \sin [80] \sin [x]+3 \csc [20]^{4} \sin [80]^{2} \sin [x] \\
& -240 \csc [20]^{4} \csc [40] \sin [80]^{2} \sin [x] \\
& -5 \csc [20]^{4} \sin [40] \sin [120] \sin [x] \\
& +\cos [3 x]\left(3 \csc [20]^{2}(24 x \cos [40]+5(-32-32 \cos 80+\sin [40]))\right. \\
& \left.-72 \cot [20] \sin [x]^{2}\right)+96 x \sin [x] \sin [2 x] \\
& +480 \csc [20]^{2} \csc [x] \sin [4 x]+3 \csc [20]^{2} \csc [x] \sin [80] \sin [4 x] \\
& -720 \csc [20]^{2} \sin [x] \sin [4 x]+36 x \csc [20]^{2} \sin [x] \sin [4 x] \\
& +1440 \cos [40] \csc [20]^{2} \sin [x] \sin [4 x] \\
& -72 x \cos [40] \csc [20]^{2} \sin [x] \sin [4 x] \\
& +\cos [x] \csc [20]^{2}(-2640+4320 \cos [40]-48 x \cos [40] \\
& -480 \cos [80]-48 x \cos [80]-36(-20+x) \cos [40-4 x] \\
& +48(-60+x) \cos [40-2 x]+480 \cos [80-2 x] \\
& +1920 \cos [2 x]-96 x \cos [2 x]-720 \cos [4 x] \\
& +36 x \cos [4 x]+720 \cos [4(10+x)]-36 x \cos [4(10+x)] \\
& -2880 \cos [2(20+x)]+48 x \cos [2(20+x)] \\
& +480 \cos [2(40+x)]-48 \sin [40]+12 \sin [40-4 x] \\
& +21 \sin [40-2 x]-6 \sin [80-2 x]-21 \sin [4 x]+10 \sin [6 x] \\
& -12 \sin [4(10+x)]+21 \sin [2(20+x)]-6 \sin [2(40+x)]))
\end{aligned}
$$

Thus the two term solution by HPM is $Y=y_{0}+y_{1}$ and the three term solution by HPM is $Y=y_{0}+y_{1}+y_{2}$.

From Table 1, Figure 1(a) and Figure 1(b), it can be seen that there is an insignificant difference between the results of two and three terms HPM solutions and exact solution. It motivates us to move onto the next studies using different boundary conditions.

Example 2: Consider the VDP equation with first Robin type boundary condition

$$
y^{\prime \prime}+u\left(y^{2}-1\right) y^{\prime}+y=0, \quad y(0)=0, \quad y^{\prime}(20)=1
$$

The Homotopy is,

$$
Y^{\prime \prime}+Y-\left(y_{0}^{\prime \prime}+y_{0}\right)+t\left(y_{0}^{\prime \prime}-y_{0}\right)+t\left[u\left(Y^{2}-1\right) Y^{\prime}\right]=0
$$

Putting,

$$
Y=Y_{0}+t Y_{1}+t^{2} Y_{2}, y_{0}=\sec [20] \sin [x],
$$

in Equation (3.1) and equating the coefficients of $t$ from both sides, we get

$$
\begin{gathered}
y_{0}^{\prime \prime}+y_{0}=0, \quad y_{0}(0)=0, \quad y_{0}^{\prime}(20)=1 \\
y_{1}^{\prime \prime}+y_{1}+u y_{0}^{2} y_{0}^{\prime}-u y_{0}^{\prime}=0, \quad y_{1}(0)=0, \quad y_{1}^{\prime}(20)=0 \\
y_{2}^{\prime \prime}+y_{2}+2 u y_{0} y_{1} y_{0}^{\prime}-u y_{1}^{\prime}+u y_{0}^{2} y_{1}^{\prime}=0, \quad y_{2}(0)=0, \quad y_{2}^{\prime}(20)=0
\end{gathered}
$$


Table 1. Relative errors for example 1 (Using $u=0.01$ ).

\begin{tabular}{|c|c|c|c|c|c|}
\hline $\mathrm{x}$ & HPM 2 terms & HPM 3 terms & Abbassi [2] & HPM 2 Error & HPM 3 Error \\
\hline 0 & 0 & 0 & 0 & 0 & 0 \\
\hline 1 & 0.92171 & 0.860729 & 0.861666 & 0.06098172 & 0.000937481 \\
\hline 2 & 0.996004 & 0.932566 & 0.931693 & 0.063437934 & 0.000873401 \\
\hline 3 & 0.154577 & 0.145325 & 0.145255 & 0.009251442 & 7.01962E-05 \\
\hline 4 & -0.82897 & -0.7828 & -0.783643 & 0.046167623 & 0.000842517 \\
\hline 5 & -1.05036 & -0.99452 & -0.993778 & 0.055838839 & 0.000746558 \\
\hline 6 & -0.30606 & -0.29088 & -0.290629 & 0.015181896 & 0.000248529 \\
\hline 7 & 0.719634 & 0.687061 & 0.687723 & 0.032572757 & 0.000661586 \\
\hline 8 & 1.083699 & 1.037556 & 1.036991 & 0.046143471 & 0.000564957 \\
\hline 9 & 0.451416 & 0.433663 & 0.433154 & 0.0177533 & 0.000509129 \\
\hline 10 & -0.5959 & -0.57511 & -0.575549 & 0.020785844 & 0.00043809 \\
\hline 11 & -1.09535 & -1.06034 & -1.059976 & 0.03500432 & 0.000364889 \\
\hline 12 & -0.58774 & -0.57069 & -0.569876 & 0.017048117 & 0.000814214 \\
\hline 13 & 0.460232 & 0.448929 & 0.433154 & 0.011303398 & 0.01577506 \\
\hline 14 & 1.085068 & 1.061946 & 1.061763 & 0.023121451 & 0.000183197 \\
\hline 15 & 0.712297 & 0.699029 & 0.697907 & 0.013267577 & 0.001122069 \\
\hline 16 & -0.31536 & -0.31084 & -0.310907 & 0.004517348 & $6.77414 \mathrm{E}-05$ \\
\hline 17 & -1.05307 & -1.04186 & -1.041802 & 0.011215634 & $5.4816 \mathrm{E}-05$ \\
\hline 18 & -0.8226 & -0.81587 & -0.814473 & 0.006727765 & 0.001397574 \\
\hline 19 & 0.164169 & 0.163462 & 0.163476 & 0.000707021 & $1.413 \mathrm{E}-05$ \\
\hline 20 & 1 & 1 & 1 & 0 & 0 \\
\hline
\end{tabular}

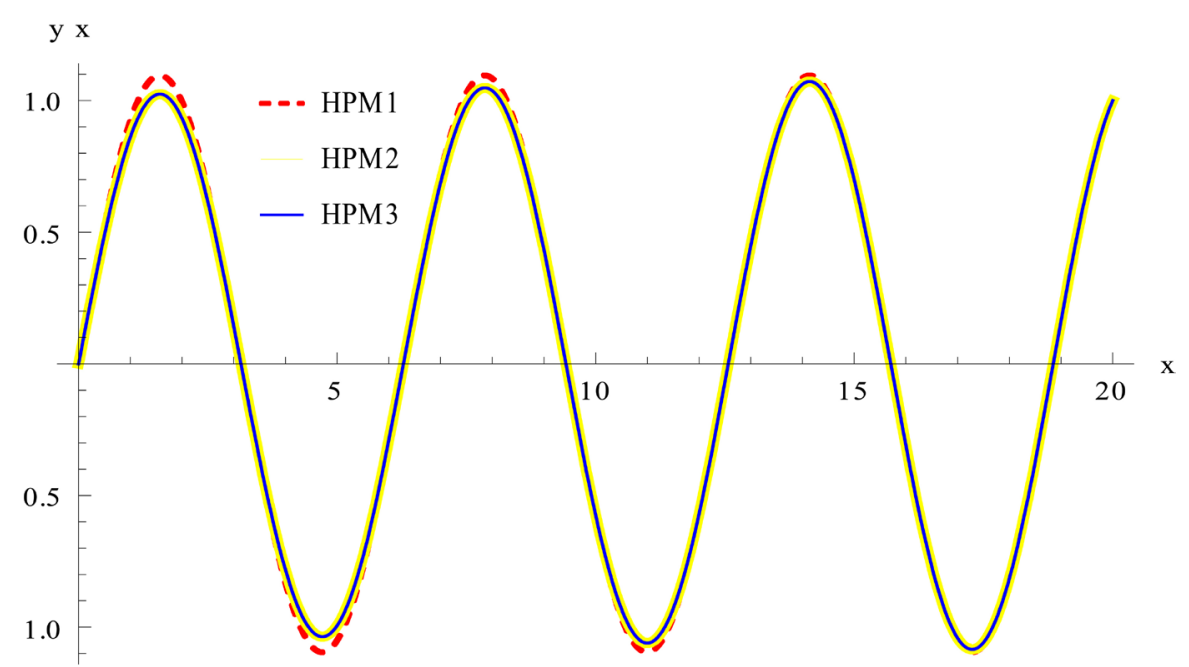

(a) 


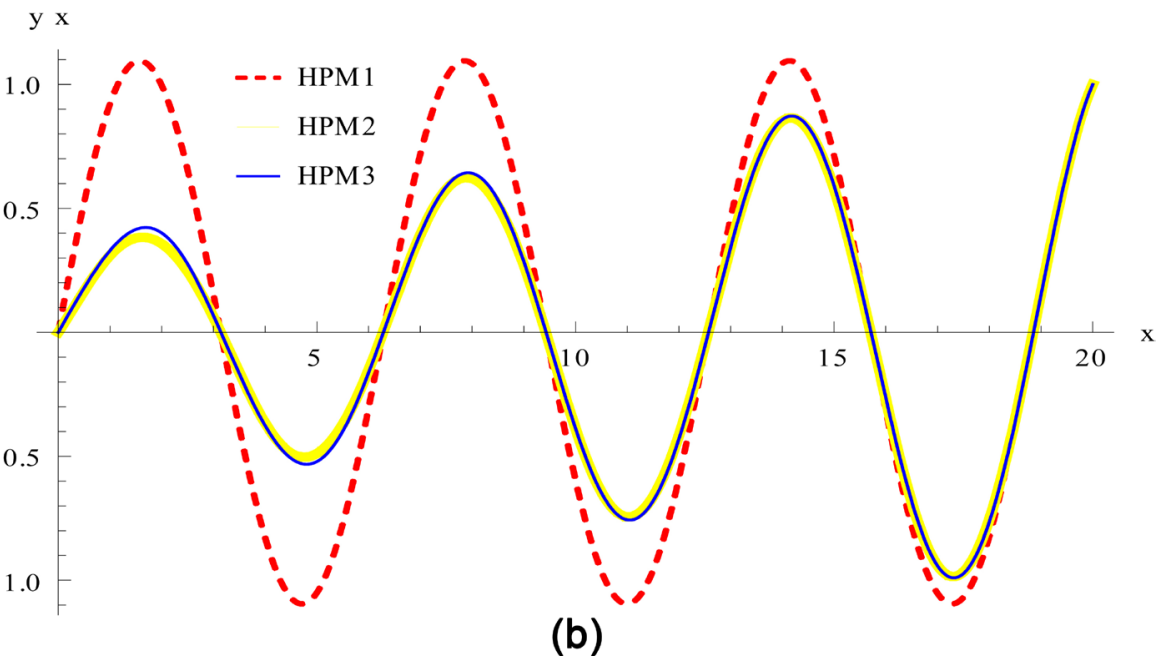

Figure 1. (a) Approximate solutions using $u=0.01$; (b) Approximate solutions using $u=$ 0.1 .

Solving we get,

$$
\begin{aligned}
& y_{0}=\sec [20] \sin [x] \\
& y_{1}=\frac{1}{32} u \sec [20]^{3} \sin [x](-160+x(4+8 \cos [40])-80 \cos [60] \sec [20] \\
& -7 \sec [20] \sin [60]+4 \cos [x] \sin [x]+\tan [20]) \\
& y_{2}=-\left(\frac{1}{6144}\right) u^{2} \sec [20] 5\left(-100 \cos [x]^{6} \sin [x]\right. \\
& -2 \cos [x]^{4}(-118+72 \cos [40]+25 \cos [2 x]) \sin [x] \\
& +(36-48 \cos [80]) \sin [x]^{3}+6(3+8 \cos [40]) \sin [x]^{5} \\
& -20 \sin [x]^{7}+12 \cos [x]^{2} \sin [x](-17+8 \cos [40] \\
& \left.-4 \cos [80]-4 \cos [60] \sec [20] \sin [x]^{2}+15 \sin [x]^{4}\right) \\
& +36(-20+x) \csc [x] \sin [2 x]^{3}-18 \cos [x]^{5}(-160 \\
& +x(4+8 \cos [40])-80 \cos [60] \sec [20]-7 \sec [20] \sin [60] \\
& +\tan [20])-\sin [x]\left(19431+48 x^{2}(1+2 \cos [80])\right. \\
& +19356 \cos [60] \sec [20]+18838 \cos [100] \sec [20] \\
& +73 \cos [140] \sec [20]-3840 \sec [20] \sin [60] \\
& +3360 \sec [20] \sin [100]-24 x \sec [20](80 \cos [20] \\
& +80 \cos [60]+80 \cos [100]+9 \sin [20]-8 \sin [60] \\
& +7 \sin [100])-9120 \sin [2 x]+81 \sin [2 x]^{2}+960 \tan [20] \\
& -1920 \cos [60] \sec [20] \tan [20]-960 \cos [100] \sec [20] \tan [20] \\
& -576 \sec [20] \sin [60] \tan [20]+220 \sec [20] \sin [100] \tan [20]
\end{aligned}
$$




$$
\begin{aligned}
& \left.+73 \sec [20] \sin [140] \tan [20]+429 \tan [20]^{2}\right) \\
& +12 \cos [x]^{3}(3 \sec [20](-80 \cos [60]+4 x \cos [60]+\sin [20] \\
& -7 \sin [60]) \sin [x]^{2}-2(-2+\cos [40])(-160+x(4+8 \cos [40]) \\
& -80 \cos [60] \sec [20]-7 \sec [20] \sin [60]+\tan [20])) \\
& +6 \cos [x](\sec [20](320 \cos [20]+80 \cos [60] \\
& -160 \cos [100]+4 x(3 \cos [20]+3 \cos [60]+4 \cos [100]) \\
& -21 \sin [20]+37 \sin [60]-14 \sin [100]) \\
& -2 \sec [20](-800 \cos [60]-80 \cos [100]+4 x(19 \cos [20] \\
& +10 \cos [60]+\cos [100])-55 \sin [60]-7 \sin [100]) \sin [x]^{2} \\
& +9 \sin [x]^{4}(-160+x(4+8 \cos [40])-80 \cos [60] \sec [20] \\
& -7 \sec [20] \sin [60]+\tan [20])))
\end{aligned}
$$

Example 3: Consider the VDP equation with second Robin type boundary condition

$$
y^{\prime \prime}+u\left(y^{2}-1\right) y^{\prime}+y=0, \quad y^{\prime}(0)=0, \quad y(20)=1
$$

The Homotopy is,

$$
Y^{\prime \prime}+Y-\left(y_{0}^{\prime \prime}+y_{0}\right)+t\left(y_{0}^{\prime \prime}-y_{0}\right)+t\left[u\left(Y^{2}-1\right) Y^{\prime}\right]=0
$$

Putting,

$$
Y=Y_{0}+t Y_{1}+t^{2} Y_{2}, \quad y_{0}=\cos [x] \sec [20],
$$

in Equation (3.1) and equating the coefficients of $t$ from both sides, we get

$$
\begin{gathered}
y_{0}^{\prime \prime}+y_{0}=0, \quad y_{0}^{\prime}(0)=0, \quad y_{0}(20)=1 \\
y_{1}^{\prime \prime}+y_{1}+u y_{0}^{2} y_{0}^{\prime}-u y_{0}^{\prime}=0, \quad y_{1}^{\prime}(0)=0, \quad y_{1}(20)=0 \\
y_{2}^{\prime \prime}+y_{2}+2 u y_{0} y_{1} y_{0}^{\prime}-u y_{1}^{\prime}+u y_{0}^{2} y_{1}^{\prime}=0, \quad y_{2}^{\prime}(0)=0, \quad y_{2}(20)=0
\end{gathered}
$$

Solving we get,

$$
\begin{aligned}
& y_{0}=\cos [x] \sec [20] \\
& y_{1}= \frac{1}{64} u \sec [20]^{3}\left(-7 \cos [x]^{2} \sin [x]+\sin [x](-1-16 \cos [40]\right. \\
&\left.+\sin [x]^{2}\right)+2 \cos [x](-160+x(4+8 \cos [40]) \\
&-80 \cos [60] \sec [20]+5 \sec [20] \sin [60]-3 \tan [20])) \\
& y_{2}=\left(\frac{1}{6144}\right) u^{2} \sec [20]^{5}\left(20 \cos [x]^{7}+6 \cos [x]^{5}(-18+16 \cos [40]\right. \\
&+15 \cos [2 x])-4 \cos [x]^{3}(33+96 \cos [40]+12 \cos [80] \\
&+\left.(-69+48 \cos [40]) \sin [x]^{2}+25 \sin [x]^{4}\right) \\
&+ 12 \cos [x]^{2} \sin [x](3 \sec [20](-160 \cos [20]-80 \cos [60] \\
&+4 x \cos [60]-3 \sin [20]+5 \sin [60]) \sin [x]^{2}
\end{aligned}
$$




$$
\begin{aligned}
& -2(4+\cos [40])(-160+x(4+8 \cos [40]) \\
& -80 \cos [60] \sec [20]+5 \sec [20] \sin [60]-3 \tan [20])) \\
& +54 \cos [x]^{4} \sin [x](-160+x(4+8 \cos [40]) \\
& -80 \cos [60] \sec [20]+5 \sec [20] \sin [60]-3 \tan [20]) \\
& -6 \sin [x](1280+36 x+560 \cos [60] \sec [20] \\
& +20 x \cos [60] \sec [20]-160 \cos [100] \sec [20] \\
& -61 \sec [20] \sin [60]+10 \sec [20] \sin [100]-12 x \sin [2 x]^{2} \\
& +4(-2+\cos [40]) \sin [x]^{2}(-160+x(4+8 \cos [40]) \\
& -80 \cos [60] \sec [20]+5 \sec [20] \sin [60]-3 \tan [20]) \\
& +3 \sin [x]^{4}(-160+x(4+8 \cos [40])-80 \cos [60] \sec [20] \\
& +5 \sec [20] \sin [60]-3 \tan [20])+49 \tan [20]) \\
& +\cos [x]\left(19455+48 x^{2}(1+2 \cos [80])+19404 \cos [60] \sec [20]\right. \\
& +19214 \cos [100] \sec [20]-7 \cos [140] \sec [20] \\
& +3840 \sec [20] \sin [60]-24 x \sec [20](80 \cos [20]+80 \cos [60] \\
& +80 \cos [100]-11 \sin [20]+8 \sin [60]-5 \sin [100]) \\
& -2400 \sec [20] \sin [100]+12(9+56 \cos [40]-4 \cos [80]) \sin [x]^{2} \\
& -6(31+48 \cos [40]) \sin [x]^{4}+100 \sin [x]^{6}+6720 \tan [20] \\
& +5760 \cos [60] \sec [20] \tan [20]-960 \cos [100] \sec [20] \tan [20] \\
& -576 \sec [20] \sin [60] \tan [20]+92 \sec [20] \sin [100] \tan [20] \\
& \left.\left.-7 \sec [20] \sin [140] \tan [20]+477 \tan [20]^{2}\right)\right)
\end{aligned}
$$

Example 4: Consider the VDP equation with Neumann type boundary condition

$$
y^{\prime \prime}+u\left(y^{2}-1\right) y^{\prime}+y=0, \quad y^{\prime}(0)=0, \quad y^{\prime}(20)=1
$$

The Homotopy is,

$$
Y^{\prime \prime}+Y-\left(y_{0}^{\prime \prime}+y_{0}\right)+t\left(y_{0}^{\prime \prime}-y_{0}\right)+t\left[u\left(Y^{2}-1\right) Y^{\prime}\right]=0
$$

Putting,

$$
Y=Y_{0}+t Y_{1}+t^{2} Y_{2}, y_{0}=-\cos [x] \csc [20],
$$

in Equation (3.1) and equating the coefficients of $t$ from both sides, we get

$$
\begin{gathered}
y_{0}^{\prime \prime}+y_{0}=0, \quad y_{0}^{\prime}(0)=0, \quad y_{0}^{\prime}(20)=1 \\
y_{1}^{\prime \prime}+y_{1}+u y_{0}^{2} y_{0}^{\prime}-u y_{0}^{\prime}=0, \quad y_{1}^{\prime}(0)=0, \quad y_{1}^{\prime}(20)=0 \\
y_{2}^{\prime \prime}+y_{2}+2 u y_{0} y_{1} y_{0}^{\prime}-u y_{1}^{\prime}+u y_{0}^{2} y_{1}^{\prime}=0, \quad y_{2}^{\prime}(0)=0, \quad y_{2}^{\prime}(20)=0
\end{gathered}
$$

Solving we get, 


$$
\begin{aligned}
& y_{0}=-\cos [x] \csc [20] \\
& y_{1}=\frac{1}{32} u \csc [20]^{3}(2 \cos [x](40-80 \cos [40]+x(-2+4 \cos [40])-3 \sin [40]) \\
&\left.+3 \cos [x]^{2} \sin [x]-\sin [x]\left(-1+8 \cos [40]+\sin [x]^{2}\right)\right) \\
& y_{2}=\left(\frac{1}{3072}\right) u^{2} \csc [20]^{5}\left(30 \cos [x]^{5}-63 \cos [x]^{2}(40-80 \cos [40]\right. \\
&+x(-2+4 \cos [40])-3 \sin [40]) \sin [x]-\cos [x]^{3}(1+84 \cos [40] \\
&\left.+25 \sin [x]^{2}\right)+3 \sin [x](520-1680 \cos [40]-640 \cos [80] \\
&+2 x(9-14 \cos [40]+8 \cos [80])-87 \sin [40]-24 \sin [80] \\
&\left.+3(40-80 \cos [40]+x(-2+4 \cos [40])-3 \sin [40]) \sin [x]^{2}\right) \\
&+\cos [x](-\csc [20](1440 \cos [20]+3840 \cos [60] \\
&-240 \cos [100]+9540 \sin [20]-9399 \sin [60]+9430 \sin [100] \\
&+24 x^{2}(\sin [20]-\sin [60]+\sin [100])+12 x(-3 \cos [20] \\
&+3 \cos [100]-80(\sin [20]-\sin [60]+\sin [100]))) \\
&\left.\left.+36(-2+7 \cos [40]) \sin [x]^{2}+25 \sin [x]^{4}\right)\right)
\end{aligned}
$$

From Figures 2-4 it can be seen that VDPDE with different boundary

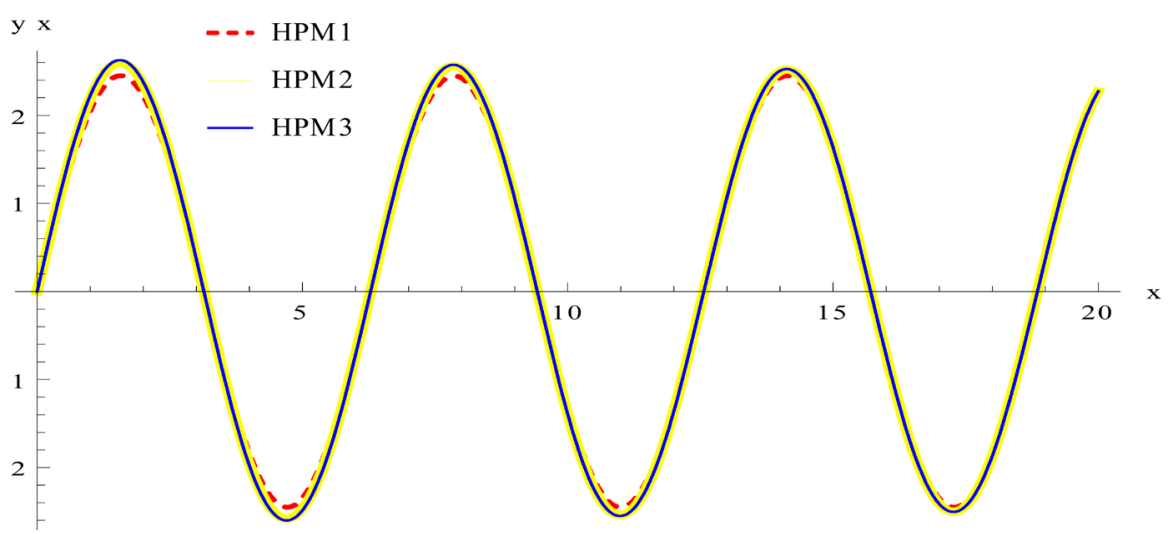

(a)

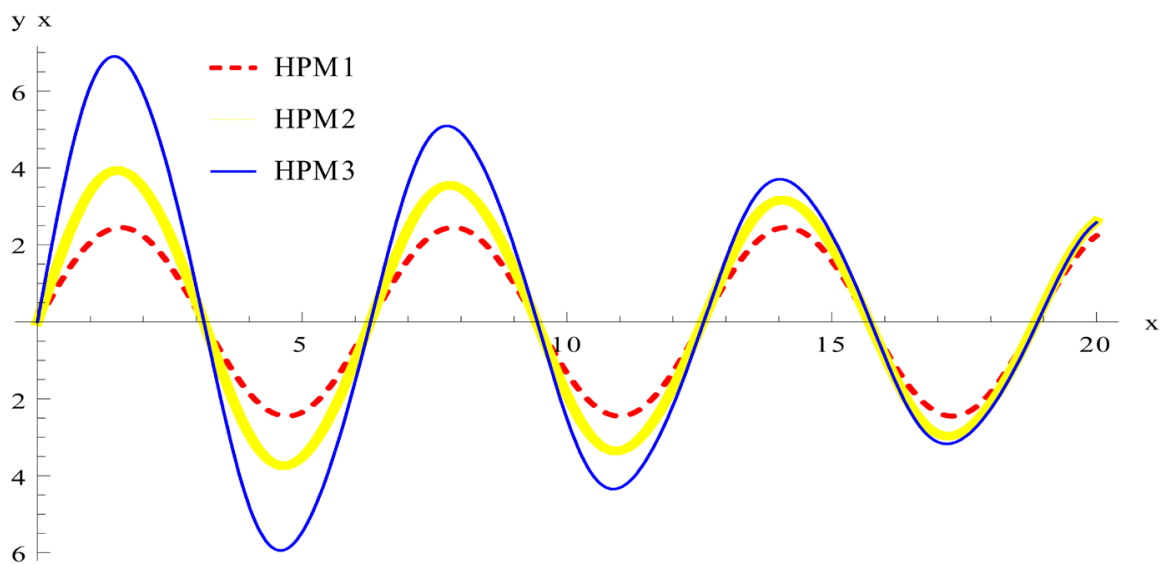

(b)

Figure 2. (a) Approximate solutions using $u=0.01$; (b) Approximate solutions using $u=0.1$. 


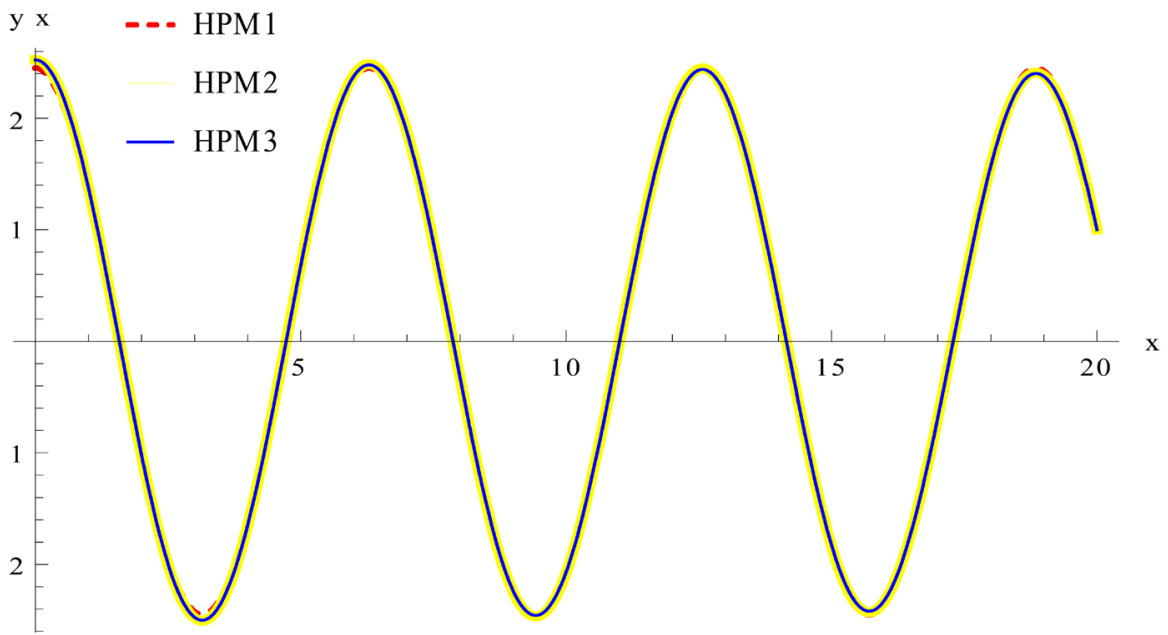

(a)

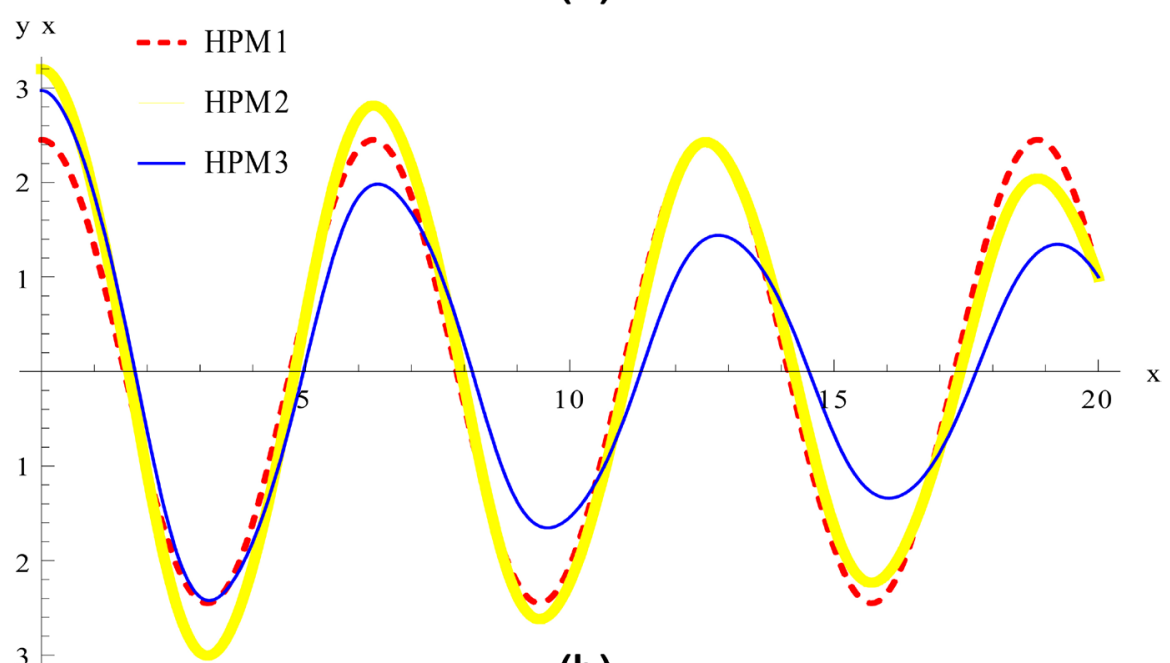

(b)

Figure 3. (a) Approximate solutions using $u=0.01$; (b) Approximate solutions using $u=$ 0.1 .

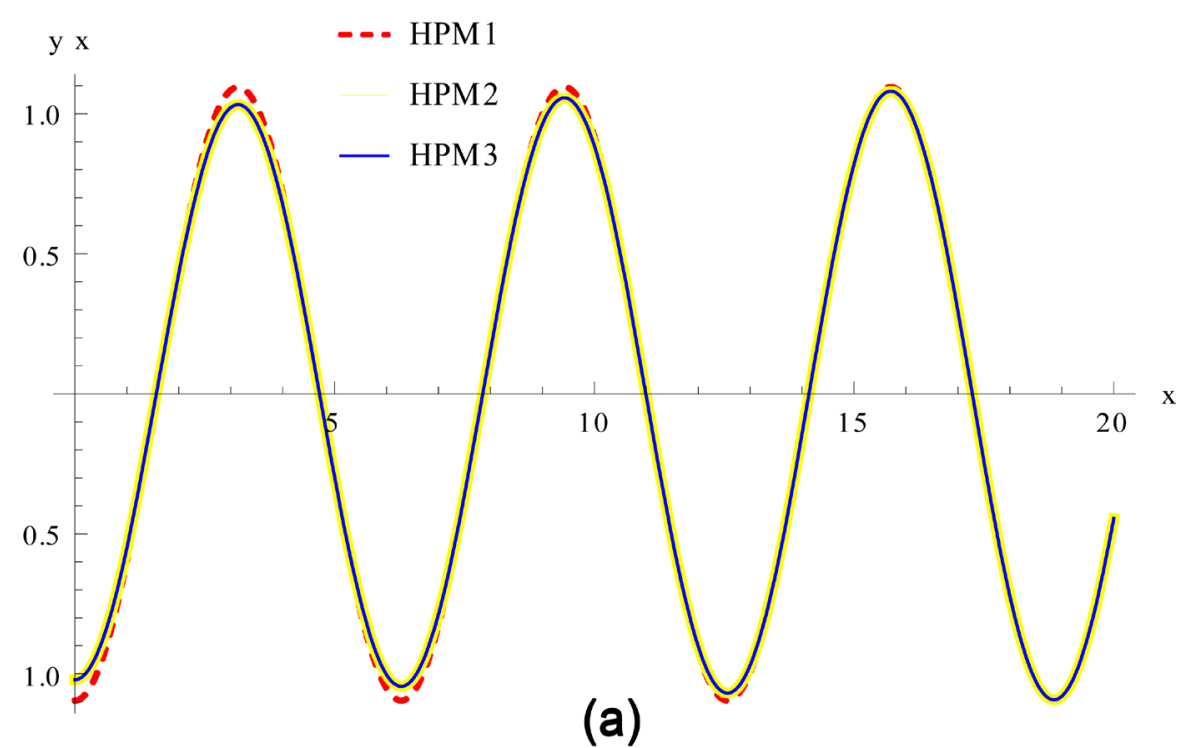




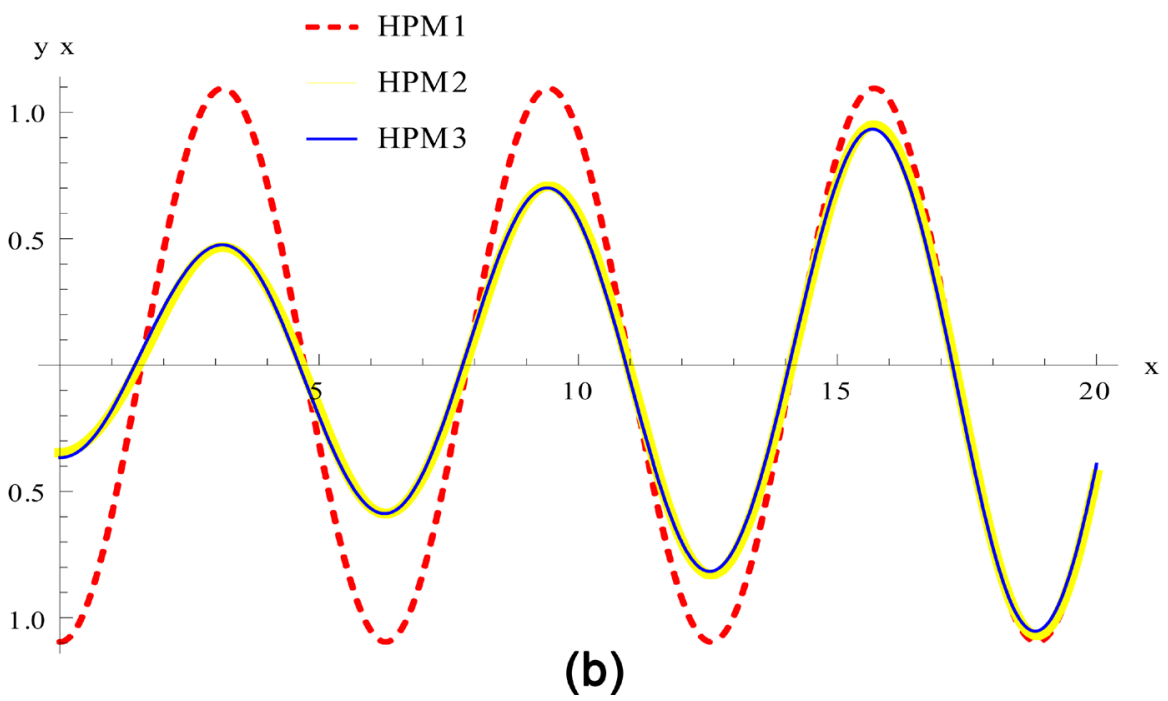

Figure 4. (a) Approximate solutions using $u=0.01$; (b) Approximate solutions using $u=0.1$.

conditions can be solved by HPM very easily. Two terms and three terms solution almost coincide. Increasing the number of terms, more accurate results can be found. The solution can be obtained by using different values of $u$.

\section{Conclusion}

In this research, HPM is applied for the solution of the Van Der Pol differential equation with different boundary conditions. One, two and three parameters HP solutions are developed and presented graphically. It is found that higher parameter shows good approximations of the analytical solution. It may conclude that HPM is a very effective technique to find the analytical solutions for highly non-linear ordinary differential equation with ICs/BCs.

\section{Conflicts of Interest}

The author declares no conflicts of interest regarding the publication of this paper.

\section{References}

[1] Buonomo, A. (1998) The Periodic Solution of Van der Pol's Equation. SIAM Journal of Applied Mathematics, 59, 156-171. https://doi.org/10.1137/S0036139997319797

[2] Abbasi, N.M. (2009) Solving the Van Der Pol Nonlinear Differential Equation Using First Order Approximation Perturbation Method.

[3] Deeba, E. and Xie, S. (2001) The Asymptotic Expansion and Numerical Verification of Van der Pol's Equation. Journal of Computational Analysis and Applications, 3, 165-171. https://doi.org/10.1023/A:1010189225921

[4] He, J.H. (1999) Homotopy Perturbation Technique. Computer Methods in Applied Mechanics and Engineering, 178, 257-262. https://doi.org/10.1016/S0045-7825(99)00018-3

[5] He, J.H. (2009) An Elementary Introduction to the Homotopy Perturbation Me- 
thod. Computers and Mathematics with Applications, 57, 410-412.

https://doi.org/10.1016/j.camwa.2008.06.003

[6] Rafiq, A., Ahmed, M. and Hussain, S. (2008) A General Approach to Specific Second Order Ordinary Differential Equations Using Homotopy Perturbation Method. Physics Letters A, 372, 4973-4976.

https://doi.org/10.1016/j.physleta.2008.05.070

[7] Chowdhury, M.S.H. and Hashim, I. (2009) Solutions of Emden-Fowler Equations by Homotopy Perturbation Method. Nonlinear Analysis. Real World Applications, 10, 104-115. https://doi.org/10.1016/j.nonrwa.2007.08.017

[8] Yıldırım, A. and Özis, T. (2007) Solutions of Singular IVPs of Lane-Emden Type by Homotopy Perturbation Method. Physics Letters A, 369, 70-76.

https://doi.org/10.1016/j.physleta.2007.04.072

[9] Saadatmandia, A., Dehghanb, M. and Eftekharia, A. (2009) Application of He's Homotopy Perturbation Method for Non-Linear System of Second-Order Boundary Value Problems. Nonlinear Analysis. Real World Applications, 10, 1912-1922. https://doi.org/10.1016/j.nonrwa.2008.02.032 